\title{
Static and optical field enhancement in metallic nanotips studied by two-photon photoemission microscopy and spectroscopy excited by picosecond laser pulses
}

\author{
S. Tsujino, ${ }^{\text {a) }}$ F. le Pimpec, J. Raabe, M. Buess, M. Dehler, E. Kirk, J. Gobrecht, and \\ A. Wrulich \\ Paul Scherrer Institut, CH-5232 Villigen-PSI, Switzerland
}

(Received 22 December 2008; accepted 12 February 2009; published online 5 March 2009)

\begin{abstract}
The authors studied the photoemission from molybdenum nanotip arrays with controlled apex curvatures by photoelectron microscopy and spectroscopy excited by 10 ps visible laser pulses under dc electric field. While two-photon photoemission microscopy demonstrates the enhancement of photoabsorption at the sharp tip apex, spatially resolved analysis of the photoelectron energy revealed anomalous energy distribution that is ascribed to the dc field enhancement and resultant barrier reduction at the tip apex. The results show the applicability of photoelectron microscopy and spectroscopy to study the distribution of dc and optical electric field enhancement in field-emitter arrays. (C) 2009 American Institute of Physics. [DOI: 10.1063/1.3095480]
\end{abstract}

A low-emittance and high-brightness electron source is advantageous for the development of compact $\mathrm{x}$-ray free electron lasers. ${ }^{1-3}$ For such applications, field-emitter array (FEA) devices equipped with a collimation gate in addition to an electron extraction gate can potentially outperform ${ }^{4,5}$ the state-of-the-art photocathode. ${ }^{6}$ So far, the realization of FEAs with large emission current in the range of several amperes with the average current density of $\sim 1 \mathrm{kA} / \mathrm{cm}^{2}$ or higher is a challenge. Some of the difficulties lie in the controllability of the sharpness and the surface quality of the nanometer-scale tip apex over the entire array of tens of thousand tips. The uniformity of the apex curvature is important ${ }^{7}$ since the Fowler-Nordheim tunneling current varies sensitively with the actual dc electric field $F_{\mathrm{dc}}$ at the apex. ${ }^{8,9} F_{\mathrm{dc}}$ is written as $\beta F_{\mathrm{av}}$, determined by the applied electric field $F_{\text {av }}$ and the dc field enhancement factor $\beta$. Since $\beta$ is approximately proportional to the inverse of the tip-apex curvature $\rho$ (Ref. 9), one finds that a factor of two variation in $\rho$ over an emitter array results in $2-5$ orders of magnitude variation in the emission current from individual emitters. Therefore, the uniformity of $\rho$ is crucial to prevent the premature failure of the device at low current level. Hence, precise characterization of the distribution of $\rho$ and $\beta$ is important for the optimization of fabrication processes. Scanning anode field emission microscopy ${ }^{10}$ is one such technique, where $\beta$ is evaluated from local field emission characteristics measured by the scanning anode probe.

In addition to dc field emission, laser triggered field emission has been extensively studied recently to generate ultrafast electron beams in needle-shaped emitters ${ }^{11-15}$ as well as in FEAs. ${ }^{16}$ In particular, single-photon photoemission triggered by 50 fs near infrared light pulses was observed in single-gate molybdenum FEAs under large $F_{\mathrm{dc}}$ in fieldemission regime with tip quantum efficiencies in the order of $10^{-2}$ (Ref. 16). For quantitative understanding and further improvement of the quantum efficiency, the impact of apex curvature on the light coupling needs to be explored. Scanning probe microscopy was used in the past to study the light

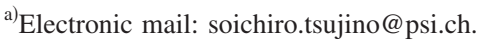

excitation in subwavelength protrusions. ${ }^{17}$ However, the light excitation at the sharp apex of field emitters is yet to be explored.

In this letter, we report an application of photoelectron emission microscopy (PEEM) to metallic FEAs for the purpose of characterizing tip uniformity and photoexcitation efficiency at the tip apex. Triggering the photoemission by tightly focused laser pulses, we observed topographydependent photoemission images. Further, spatially resolved analysis of photoelectron energy revealed tip-apex dependent anomalous energy distribution.

The metallic FEA samples used for this work were fabricated by a molding technique, Fig. 1 . The samples consist of pyramidal shaped molybdenum emitters with a base size of 1-3 $\mu \mathrm{m}$ and aligned with a period of 5-10 $\mu \mathrm{m}$. Details of the fabrication procedure are described elsewhere. ${ }^{18} \mathrm{We}$ studied two emitter structures having a base size of $3 \mu \mathrm{m}$ square and height of $\sim 2 \mu \mathrm{m}$ aligned with $10 \mu \mathrm{m}$ period. In Fig. 1 we show the scanning electron microscope (SEM) images of single emitters from the two samples: sample A [Figs. 1(a) and 1(b)] with the apex diameter of $\sim 100 \mathrm{~nm}$
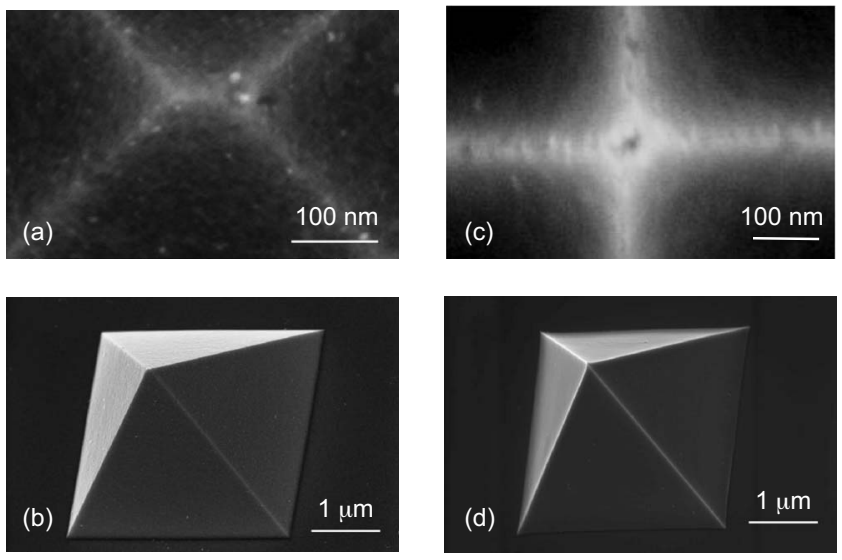

FIG. 1. SEM images of two field emitters. [(a) and (b)] show sample A, nonsharpened emitter with $\sim 100 \mathrm{~nm}$ apex curvature, and [(c) and (d)] show sample B, sharpened emitter with (1-5) nm apex curvature, respectively. 

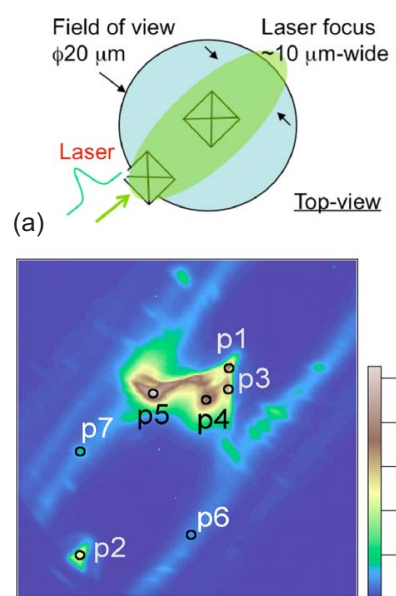

(b)

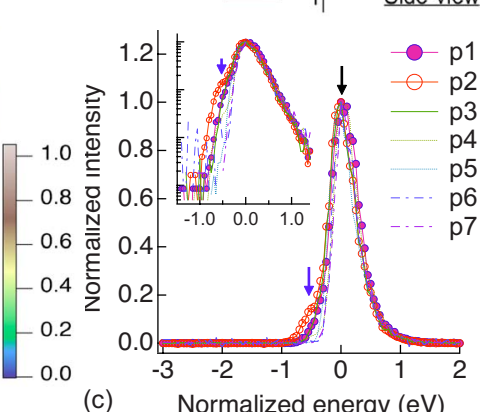

FIG. 2. (Color online) (a) Schematics of the photoemission electron microscopy and spectroscopy of field emitters excited by pulsed laser. (b) PEEM image of sample A when $4.5 \mathrm{~nJ}$ laser pulses irradiate the structure from the bottom left to the top-right direction. (c) Normalized spatially resolved photoelectron energy distribution. The inset shows the same spectra in log scale. The spectra were overlaid for clarity.

and sample B [Figs. 1(c) and 1(d)] with the apex diameter in the range of $(1-5) \mathrm{nm}$.

The laser triggered PEEM experiment was performed at the surface/interface microscopy beamline of the Swiss Light Source at Paul Scherrer Institut. The experimental geometry is depicted in Fig. 2. A FEA sample was mounted on a copper holder covered with an electrostatic shield and loaded in the microscope with background pressure of $10^{-9}$ mbar. The sample was placed in front of the electron objective lens separated by $(2.0 \pm 0.1) \mathrm{mm}$ and was dc biased at $-20 \mathrm{kV}$, corresponding to $F_{\text {av }}$ of $(10 \pm 0.5) \mathrm{MV} / \mathrm{m}$. Photoelectrons were generated by irradiation with the second harmonic output of a mode-locked neodymium-doped yttrium aluminum garnet laser with pulse energy up to $\sim 5 \mathrm{~nJ}$, pulse width of $10 \mathrm{ps}$ (full width at half maximum), repetition time of $16 \mathrm{~ns}$, and wavelength of $532 \mathrm{~nm}$ corresponding to a photon energy $\hbar \omega$ of $2.33 \mathrm{eV}$. Thus, $\hbar \omega$ is slightly larger than one half of the work function of the molybdenum (100) surface, which is equal to $\sim 4.5 \mathrm{eV}$. The incident angle of the light was $\sim 75^{\circ}$ from the sample surface normal direction and the light polarization was fixed within the incident plane (p-polarization). The laser pulses were focused to an $\sim 10 \mu \mathrm{m}$ wide beam on the sample. An electron objective lens collected the photoelectrons, which were subsequently energy analyzed and detected by a phosphor screen after amplification by a multichannel plate. The resolution of the energy analyzer of the PEEM is in the range of $(0.1-0.3) \mathrm{eV}$.

In Fig. 2(b), we show the PEEM image of sample A. It was acquired with a $20 \mu \mathrm{m}$ field of view and by integrating over $6 \mathrm{eV}$ of the scanned energy range. Two emitters, one at the center and another at the bottom left (only the apex), were imaged by the spatial contrast of the photoelectron intensity. The photoelectrons were excited via two-photon absorption (TPA) process. This was confirmed by the quadratic increase in the photoelectron intensity when the pulse energy was varied between 1 and $5 \mathrm{~nJ}$ (not shown). In addition to the facet of the pyramidal shaped emitter (marked as p4) facing the laser irradiation, the sharp parts of the emitters such as the apexes (p1 and p2), the wedges, and along the

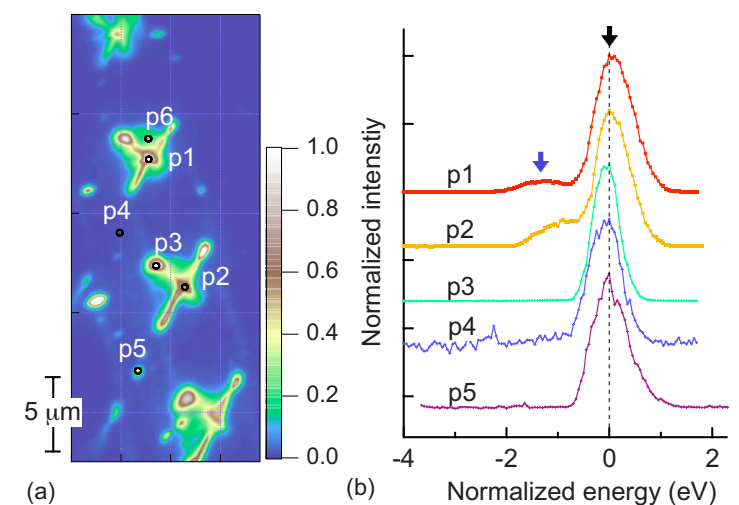

FIG. 3. (Color online) (a) PEEM image of sample B having the emitter apex curvature in the order of (1-5) nm. (b) Normalized spatially resolved photoelectron energy spectra when the emitters were excited by $3 \mathrm{~nJ}$ pulses. The spectra were shifted vertically for clarity.

edges at the bottom (p3 and p5) emit high intensity of photoelectrons. The photoelectron intensities from the apex are comparable to the emitter facets within the spatial resolution of the experiment and are an order of magnitude higher than those from the flat part of the sample (p6 and p7). The observation of these bright spots shows that the light excitation is enhanced at sharp locations of the emitters, especially at the emitter apexes (p1 and p2).

Such an enhanced laser excitation is further evidenced by the result of sample B, where the radius of curvature at the apex, as well as at the edges of the emitters, was more than a factor of 20 smaller than sample A, Fig. 3(a). In this experiment, the laser irradiates the emitters from top left toward bottom-right direction, and the PEEM image was acquired with a $50 \mu \mathrm{m}$ diameter field of view. Comparison of Fig. 3(a) to Fig. 2(b) with respect to the photoemission contrast between the emitter apexes/edges and, e.g., the emitter facets shows that the light excitation efficiency is enhanced by decreasing the radius of curvature of the protrusions.

From the PEEM image of sample B [Fig. 3(a)], we found that TPA at apex (p1) is higher than that at the facet (p6) by a factor of 10. Since TPA at the emitter apex is proportional to $\left(\eta F_{\mathrm{op}}\right)^{4}$, where $F_{\text {op }}$ is the optical electric field and $\eta$ is the enhancement factor of $F_{\mathrm{op}}, \eta$ equal to $\sim 1.8$ is evaluated from the observed photoemission intensity ratio between $\mathrm{p} 1$ and $\mathrm{p} 6$. This result indicates that single-photon absorption at a nanometer scale emitter apex, which is proportional to $\left(\eta F_{\mathrm{op}}\right)^{2}$, e.g., as reported in Ref. 16, is a factor of $\sim 3$ more efficient than at flat surface. We note that $\eta \sim 1.8$ is of the same order of magnitude as the value of $\sim 4$ reported by Martin et al. ${ }^{19}$ but largely deviates from the value of 120 evaluated for a tungsten needle. ${ }^{20}$ Detailed analysis of the observed $\eta$ and its comparison with theory are the subject of the future research and beyond the scope of this letter.

Finally, we discuss the photoelectron energy distributions. In Figs. 2(c) and 3(b), we show spectra for samples A and $\mathrm{B}$, respectively. Because of the highly nonplanar nature of the sample, the spectra shift monotonically within the field of view (by $\sim 0.4 \mathrm{eV}$ from right to left in the case of sample A). To compare the shape of distributions from different surface locations, we therefore lined up the peak maxima of the spectra to zero and normalized the maximum of every curve as 1 . The slopes of the high-energy tails are similar for all the positions for each sample. However, the low-energy tails are 
TABLE I. Summary of the emitter apex curvature $\rho$, the calculated and experimental dc field enhancement factors $\beta^{(\text {calc })}$ and $\beta^{(\exp )}$, and the energy difference $\Delta W$ between the onset of the low-energy shoulders at the emitter apex and the main TPA peak of the surroundings at $0 \mathrm{eV}$.

\begin{tabular}{lcccc}
\hline \hline & $\begin{array}{c}\rho \\
\text { Sample }\end{array}$ & $\beta^{\text {(calc) }}$ & $\begin{array}{c}\Delta W \\
(\mathrm{eV})\end{array}$ & $\beta^{(\exp )}$ \\
\hline A & $40 \pm 15$ & $19 \pm 4$ & $0.55 \pm 0.10$ & $32 \pm 9$ \\
B & $3 \pm 2$ & $(1.5 \pm 0.7) \times 10^{2}$ & $1.3 \pm 0.5$ & $(1.6 \pm 1.0) \times 10^{2}$ \\
\hline \hline
\end{tabular}

position dependent: at the emitter apex, additional shoulders were observed at $-0.5 \mathrm{eV}$. The low-energy shoulders were also observed in sample B (Fig. 3) at the emitter apex but at energies of $-(1-1.5) \mathrm{eV}$. Cinchetti et al. ${ }^{21}$ reported photoemission from local inhomogeneities (hot spots) on a $\mathrm{Cu}$ surface shifted to low energy by $\sim 0.5 \mathrm{eV}$ and ascribed those to reduced work functions in small $\mathrm{Cu}$ clusters. The hot spots on $\mathrm{Cu}(001)$ surface were studied by Georgiev et al. in detail. ${ }^{22}$ However, we did not observe such low-energyshifted features either at the side edges of the emitters or at the hot spots on the flat part of the samples p6 and p7 in Fig. 2(b) and p4 and p5 in Fig. 3.

We consider that the observed low-energy shoulders indicate the two-photon photoemission from the emitter apex with reduced barrier height. ${ }^{23}$ Because of the dc field enhancement, the barrier height reduction at the emitter apex is enhanced compared to the surroundings by the amount $\Delta E$ given by $\sqrt{e^{3} F_{\text {av }} /\left(4 \pi \varepsilon_{0}\right)}\{\sqrt{\beta}-1\}$ with $\varepsilon_{0}$ as the vacuum permittivity and $e$ as the elementary charge. ${ }^{24}$ Therefore, by equating $\Delta E$ with the energy difference $\Delta W$ between the onset of the observed low-energy shoulders at the emitter apex and the main TPA peak at $0 \mathrm{eV}$ of the surroundings and using $F_{\text {av }}$ of $(10 \pm 0.5) \mathrm{MV} / \mathrm{m}$ applied to FEAs, we evaluated the experimental dc field enhancement factors $\beta^{(\exp )}$, as summarized in Table I, together with the theoretical values $\beta^{\text {(calc) }}$ (Ref. 25). A good agreement of theory with experiment is found. The large distribution of the low-energy onset of the photoelectron energy distribution in sample B (Fig. 3) compared to that of sample A (Fig. 2) is ascribed to the higher sensitivity of sample B to $F_{\mathrm{av}}$ because of the larger $\beta$ as well as larger fractional variation in $\beta$ because of the small emitter radius.

In summary, we applied laser-excited PEEM and spectroscopy to study the field-enhancement properties of molybdenum FEAs. We showed a potential use of two-photon photoemission microscopy and spectroscopy as a noninvasive measurement method of the distribution of the electric field enhancement factors for dc and optical frequencies.

The authors acknowledge $\mathrm{H}$. Sehr for his help in the process development, F. Nolting for his support to make
PEEM available in the experiment, and J.-Y. Raguin for helpful discussions in the simulation of the dc fieldenhancement factor.

${ }^{1}$ J. Rossbach, E. L. Saldin, E. A. Schneidmiller, and M. V. Yurkov, Nucl. Instrum. Methods Phys. Res. A 374, 401 (1996).

${ }^{2}$ Z. Huang and K.-J. Kim, Phys. Rev. ST Accel. Beams 10, 034801 (2007).

${ }^{3}$ R. J. Bakker, A. Adelmann, A. Anghel, M. Dehler, R. Ganter, S. C. Leemann, K. Li, M. Pedrozzi, J.-Y. Raguin, L. Rivkin, V. Schlott, F. Wei, and A. Wrulich, Proceedings of the 28th International Free Electron Laser Conference, Berlin, Germany, 2006 (unpublished).

${ }^{4}$ S. C. Leemann, A. Streun, and A. F. Wrulich, Phys. Rev. ST Accel. Beams 10, 071302 (2007).

${ }^{5}$ M. Dehler, A. Candel, and E. Gjonaj, J. Vac. Sci. Technol. B 24, 892 (2006).

${ }^{6}$ K. Abrahamyan, J. Bähra, J. P. Carneirob, K. Flöttmannb, J. H. Hana, M.v. Hartrottc, M. Krasilnikova, D. Lipkaa, V. Miltcheva, A. Oppelta, B. Petrossyana, D. Posea, D. Richterc, S. Schreiberb, L. Staykova, and F. Stephan, Nucl. Instrum. Methods Phys. Res. A 558, 249 (2006).

${ }^{7}$ P. R. Schwoebel, C. A. Spindt, C. E. Holland, and J. A. Panitz, J. Vac. Sci. Technol. B 19, 582 (2001).

${ }^{8}$ R. G. Forbes and J. H. B. Deane, Proc. R. Soc. London, Ser. A 463, 2907 (2007).

${ }^{9}$ I. Brodie and S. A. Spindt, in Advances in Electronics and Electron Physics (Academic, New York, 1992), Vol. 83, p. 14.

${ }^{10}$ L. Nilsson, O. Groening, P. Groening, O. Kuettel, and L. Schlapbach, J. Appl. Phys. 90, 768 (2001).

${ }^{11}$ C. A. Brau, Nucl. Instrum. Methods Phys. Res. A 407, 1 (1998).

${ }^{12}$ P. Hommelhoff, Y. Sortais, A. Aghjani-Talesh, and M. A. Kasevich, Phys. Rev. Lett. 96, 077401 (2006).

${ }^{13}$ C. Ropers, D. R. Solli, C. P. Schulz, C. Lienau, and T. Elsaesser, Phys. Rev. Lett. 98, 043907 (2007).

${ }^{14}$ B. Barwick, C. Corder, J. Strohaber, N. Chandler-Smith, C. Uiterwaal, and H. Batellan, New J. Phys. 9, 142 (2007).

${ }^{15}$ R. Ganter, R. Bakker, C. Gough, S. C. Leemann, M. Paraliev, M. Pedrozzi, F. Le Pimpec, V. Schlott, L. Rivkin, and A. Wrulich, Phys. Rev. Lett. 100, 064801 (2008).

${ }^{16}$ S. Tsujino, P. Beaud, E. Kirk, T. Vogel, H. Sehr, J. Gobrecht, and A. Wrulich, Appl. Phys. Lett. 92, 193501 (2008).

${ }^{17}$ J. St. Grafström, Appl. Phys. (Berlin) 91, 1717 (2002).

${ }^{18}$ E. Kirk, S. Tsujino, T. Vogel, K. Jefimovs, J. Gobrecht, and A. Wrulich (unpublished).

${ }^{19}$ Y. C. Martin, H. F. Hamann, and H. K. Wickramasinghe, J. Appl. Phys. 89, 5774 (2001).

${ }^{20}$ B. Gault, F. Vurpilot, A. Bostel, A. Menand, and B. Deconihout, Appl. Phys. Lett. 86, 094101 (2005).

${ }^{21}$ M. Cinchetti, A. Oelsner, G. H. Fechner, H.-J. Elmers, and G. Schönhense, Appl. Phys. Lett. 83, 1503 (2003).

${ }^{22}$ N. Georgiev, D. Martinotti, and H.-J. Ernst, Phys. Rev. B 75, 085430 (2007)

${ }^{23}$ Because of the limited spatial resolution, the PEEM signal from $\mathrm{p} 1$ and $\mathrm{p} 2$ are superpositions of the emission from the emitter apexes (the low-energy shoulder) and the immediate vicinity (the peak at $0 \mathrm{eV}$ ).

${ }^{24}$ K. L. Jensen, in Vacuum Microelectronics, edited by W. Zhu (Wiley, New York, 2001), p. 57.

${ }^{25}$ We calculated $\beta^{\text {(calc) }}$ by the finite-element method with an adaptive meshing. We approximated our pyramidal shaped emitter by a cone-shaped emitter having same height and base size terminated by spherical surface with a radius of $\rho$ at the apex. 